\title{
Visual performance of myopia control soft contact lenses in non-presbyopic myopes
}

This article was published in the following Dove Press journal:

Clinical Optometry

\author{
Jennifer Sha' \\ Daniel Tilia ${ }^{1,2}$ \\ Jennie Diec' \\ Cathleen Fedtke ${ }^{1,2}$ \\ Nisha Yeotikar' \\ Monica Jong ${ }^{1,2}$ \\ Varghese Thomas' \\ Ravi C Bakaraju ${ }^{1,2}$
}

'Brien Holden Vision Institute, Sydney, NSW, Australia; ${ }^{2}$ School of Optometry and Vision Science, University of New South Wales, Sydney, NSW, Australia
Correspondence: Ravi C Bakaraju University of New South Wales Sydney, Level 5, Rupert Myers Building, North Wing, Gate 14 Barker Street, NSW 2052, Australia

Tel +6I 293857516

Fax +6I 29385740 I

Email r.bakaraju@brienholdenvision.org
Purpose: To compare the visual performance of soft contact lenses reported to reduce myopia progression.

Methods: In a double-blind, randomized, crossover trial, 30 non-presbyopic myopes wore MiSight ${ }^{\mathrm{TM}}$, center-distance Proclear ${ }^{\mathbb{B}}$ Multifocal (+2.00 D add), and two prototype lenses for 1 week each. High- and low-contrast visual acuities at $6 \mathrm{~m}$, and 70 and $40 \mathrm{~cm}$; stereopsis at $40 \mathrm{~cm}$; accommodative facility at $33 \mathrm{~cm}$; and horizontal phoria at $3 \mathrm{~m}$ and $33 \mathrm{~cm}$ were measured after 1 week. Subjective performance was assessed on a numeric rating scale for vision clarity, lack of ghosting, vision stability, haloes, overall vision satisfaction, and ocular comfort. Frequency of eye-strain symptoms and willingness to purchase lenses were also reported with categorical responses. Participants reported wearing times (total and visually acceptable). Linear mixed models and chi-square tests were employed in analysis with level of significance set at $5 \%$. Theoretical optical performance of all lenses was assessed with schematic myopic model eyes $(-1.00,-3.00$, and $-6.00 \mathrm{D})$ by comparing the slope of the edge spread function (ESF), an indicator for optical performance/resolution and the blur patch size of the line spread function, an indicator for contrast, between the lenses.

Results: Proclear Multifocal and MiSight provided the best distance acuities. However, the prototype lenses were rated significantly higher for many subjective variables, and there were no subjective variables where commercial lenses were rated significantly higher than the prototypes. Theoretical optical performance showed steeper slopes of the ESF and greater blur patch sizes of the LSP with commercial lenses, supporting the clinical findings of better visual acuities but reduced subjective performance. Participants wore prototypes longer and reported their vision acceptable for longer each day compared to MiSight. Both prototypes had the highest willingness-to-purchase rate.

Conclusions: The prototypes were better tolerated by myopes compared to the commercial soft contact lenses currently used for slowing myopia progression.

Keywords: accommodation, theoretical optical performance, extended depth of focus

\section{Introduction}

Myopia is a growing problem, with an estimated $22.9 \%$ and $2.7 \%$ of the world's population currently experiencing myopia and high myopia, respectively. ${ }^{1}$ These figures are estimated to grow to $49.8 \%$ and $9.8 \%$, respectively, by $2050 .^{1}$

Myopia, and in particular high myopia (usually defined as a refractive error of at least $-5.00 \mathrm{D}$ ), is associated with increased rates of ocular pathology such as retinal detachment, myopic macular degeneration, glaucoma, and cataracts. ${ }^{2,3}$ Not only are these conditions of concern to the individual, but they also place an economic burden 
on society. Affected patients require complex treatments and frequent visits to eye-care practitioners, and even in the absence of other pathology, best-corrected vision can be reduced because of the changes in ocular anatomy associated with high myopia. ${ }^{4-10}$ These factors can lead to a decrease in productivity, independence, and quality of life. ${ }^{4}$

Optical strategies to reduce the progression of myopia in children have been of rising interest over the past several decades. One method that has emerged is the use of multiplepower soft contact lenses (CLs), which are relatively simple for most eye-care practitioners to fit compared to other strategies such as orthokeratology. Being soft CLs, they are also cosmetically acceptable compared to spectacles, can improve quality of life, ${ }^{11}$ and are generally able to be handled and worn by children. ${ }^{12}$

Soft CLs used for myopia control based on the peripheral hyperopia theory have a distance portion of the lens to correct myopia, surrounded by optics that induce myopic defocus in the peripheral retina. Examples of such lenses include centerdistance Proclear ${ }^{\circledR}$ Multifocal (CooperVision, Pleasanton, CA, USA) and a novel lens type used in a study by Sankaridurg et al. The former was reported to slow myopia progression by $51 \%$ over 2 years, ${ }^{13}$ and the latter by $34 \%$ over 1 year. ${ }^{14}$ Bifocals used for myopia control include concentric rings of alternating power around the center-distance zone, examples of which include MiSight ${ }^{\mathrm{TM}}$ (CooperVision), ${ }^{15}$ and the novel DISC lens (Centre for Myopia Research, School of Optometry, The Hong Kong Polytechnic University, Kowloon, Hong Kong). ${ }^{16}$ The former was reported to slow myopia progression by $37 \%$ over 10 months $^{17}$ and $59 \%$ over 3 years, ${ }^{18}$ and the latter by $25 \%$ over 2 years. ${ }^{16}$ Additionally, Acuvue ${ }^{\circledR}$ Bifocal (Johnson \& Johnson Vision Care, Jacksonville, FL, USA) CLs were shown to slow myopia progression by $72 \%$ over 1 year in myopic children with eso fixation disparity at near. ${ }^{19}$

Two novel prototype extended-depth-of-focus (EDOF, Brien Holden Vision Pty Ltd., Sydney, NSW, Australia) CLs being tested on children living in China ${ }^{20}$ do not fall under conventional zonal bifocal or aspheric multifocal design. The optical designs of the EDOF lenses utilize selective manipulation of higher-order aberrations to achieve EDOF for the wearer, providing good vision at all customary viewing distances while minimizing unwelcomed visual compromises like ghosting and haloes commonly associated with conventional simultaneous-image multifocal designs. Additionally, visual performance with these lenses is intended to be relatively pupil neutral and relatively independent of the individual's inherent ocular aberrations. The optical design can be described to have a nonmonotonic, aperiodic, refractive power profile across its optic zone diameter (Figure 1). This design in conjunction with the optics of the wearer's eye provides an image on the retina that remains above an acceptable retinal image quality threshold continuously across a range of visual distances. These lenses are hypothesized to slow myopia progression by producing a global retinal image quality that degrades for all points behind the retina, thus acting as a stop signal for myopia progression..$^{20}$ The two EDOF lenses slowed myopia progression by $34 \%$ and $36 \%$ compared to single-vision CLs at the 6-month visit, ${ }^{20}$ and by $27 \%$ and $33 \%$ at the 12 -month visit. ${ }^{21}$

Visual performance of MiSight and Proclear Multifocal in non-presbyopic adults has been reported previously. ${ }^{22-24}$ These studies showed that even though high-contrast visual acuities were good, subjective performance was reduced compared to habitual or single-vision correction. ${ }^{22-24} \mathrm{Good}$ subjective performance is desirable in a myopia control lens to promote full-time lens wear. Indeed, Lam et al and Sankaridurg et al found that myopia progression was inversely proportional to CL wearing time. ${ }^{16,21}$

In the current study, we compare the visual performance and wearability of novel EDOF prototype lenses to that of MiSight and Proclear Multifocal in non-presbyopic adults. In addition, optical modeling of the test CLs used in conjunction with an appropriate schematic myopic model eye resulted in line and edge spread functions (LSF and ESF) that were used to explain the differences observed in the clinic.

\section{Methods Design}

This was a prospective, double-blind, crossover, randomized, 1-week dispensing clinical trial conducted at the Clinical Research and Trials Centre (CRTC) of the Brien Holden Vision Institute in Sydney, Australia, which commenced in March 2016 and concluded in January 2017. A Human Research Ethics Committee (Bellberry, Adelaide, SA, Australia) approved the clinical trial. Written informed consent was obtained from each participant before commencing any study procedures. The clinical trial conformed to the principles of the Declaration of Helsinki. The trial was registered with ANZCTR (ACTRN12615000652572) and ClinicalTrials. gov (NCT02484586).

\section{Participants}

The inclusion criteria for the study were as follows: aged between 18 and 35 years, distance high-contrast visual acuity (HCVA) correctable to at least 20/40 in each eye with study CLs, astigmatism of $\leq 1.00 \mathrm{DC}$, a spherical equivalent refraction between -6.00 and $-0.50 \mathrm{D}$, and having good ocular health to not preclude safe CL wear. Participants were also required to have no systemic conditions that would affect 

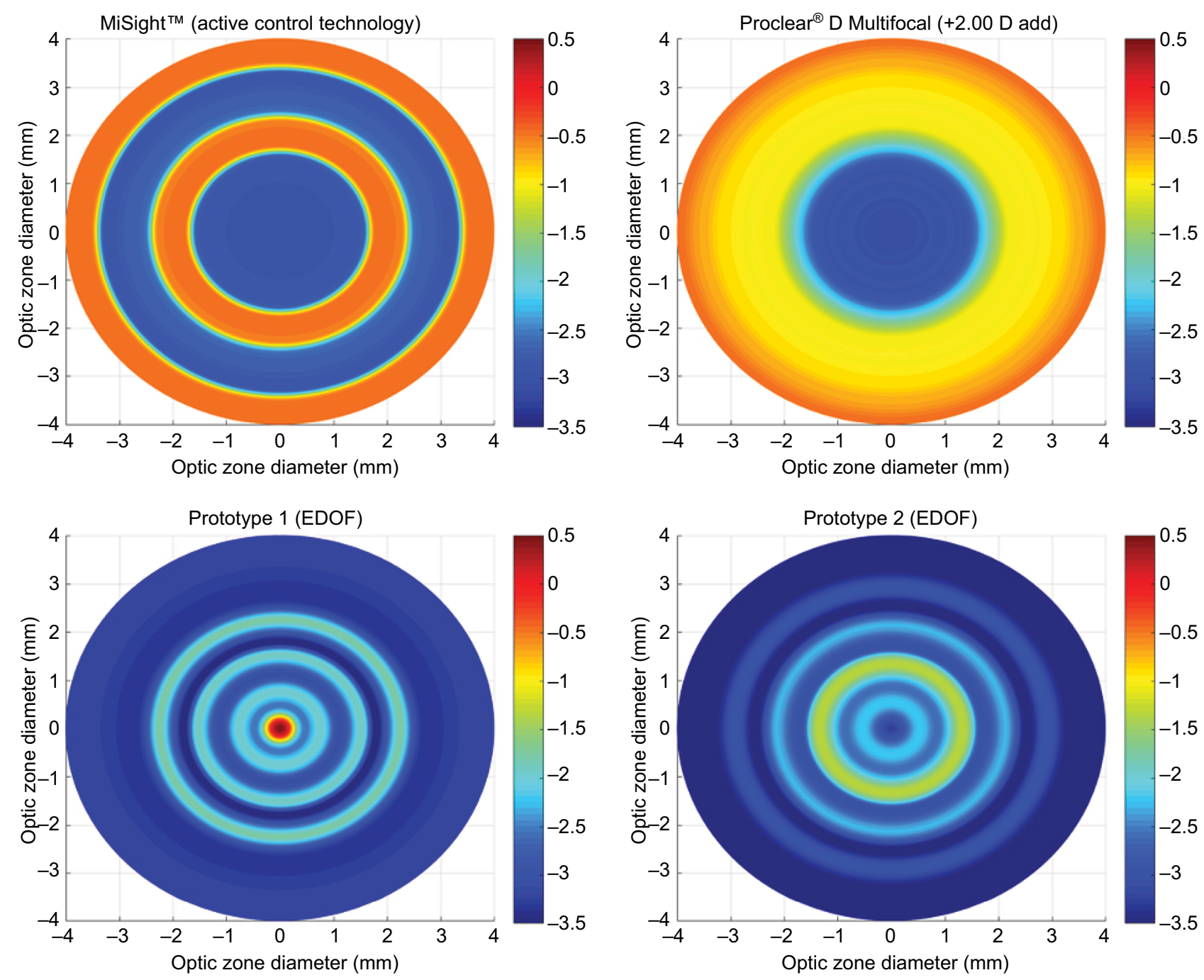

Figure I Power profiles of the study lenses ( $-3.00 \mathrm{D}$ label power).

Note: Units of the color scale are in diopters (D).

Abbreviation: EDOF, extended-depth-of-focus.

ocular health or lens wear such as diabetes or autoimmune conditions. The enrolled participants were a mix of both experienced and non-CL wearers. The latter went through a 1-week period of lens adaptation with commercial singlevision CLs for lens handling training.

\section{Baseline procedures}

A baseline visit determined the participants' subjective distance refraction, stereopsis, distance and near horizontal phoria, and monocular accommodative facility. All baseline procedures were performed under photopic conditions (350-400 lux).

Subjective distance refraction was performed using standard optometric techniques. HCVA was measured using a Test Chart 2000 Pro (Thompson Software Solutions, Hertfordshire, UK) at $6 \mathrm{~m}$, and a high-contrast black-onwhite ETDRS 2000 Series Logarithmic Visual Acuity Chart (Precision Vision, Woodstock, IL, USA) at $40 \mathrm{~cm}$.

The participant wore the full-distance subjective refraction in a trial frame for all subsequent measurements. Stereopsis was measured at $40 \mathrm{~cm}$ using the Random Dot 3 LEA SYMBOLS ${ }^{\circledR}$ Stereoacuity Test (Vision Assessment Corporation, Elk Grove Village, IL, USA). Phorias were measured using Howell-Prentice Distance and Near Cards (Cyclopean Design, Heathmont, VIC, Australia) at $3 \mathrm{~m}$ and $33 \mathrm{~cm}$, respectively. Monocular accommodative facility was measured with \pm 2.00 D flippers while viewing a $0.2 \log$ MAR size paragraph on a black-on-white MNREAD chart (Precision Vision) at $33 \mathrm{~cm}$ with the left eye occluded.

\section{Contact lenses}

Test lenses were prototype CLs manufactured in polyhydroxyethylmethacrylate (poly-HEMA; Pegavision, Taoyuan City, Taiwan) (58\% water content) with base curve 
and diameter 8.5 and $14.2 \mathrm{~mm}$, respectively. Two different designs were tested, named Prototypes 1 and 2.

Control lenses comprised Proclear Multifocal (centerdistance, +2.00 D add, omafilcon B, CooperVision) and MiSight (approximately $+2.50 \mathrm{D}$ add [Figure 1], omafilcon A, CooperVision).

Lenses were worn on a daily-wear basis for 1 week (minimum 5 days), for a minimum of 6 hours per day and disposed daily. Initial lens powers were chosen based on participants' spherical equivalent subjective distance refraction. Power profiles for all study lenses based on a $-3.00 \mathrm{D}$ label power, as measured with the NIMO TR1504 (Lambda-X, Nivelles, Belgium), are shown in Figure 1.

\section{Masking procedure}

Investigators at the CRTC were divided into two groups, unmasked and masked observers. Unmasked observers performed fitting visits during which the assigned lens (allocated through a simple randomization scheme generated through http://randomization.com) was fitted to the participant. Approximately $10 \mathrm{~min}$ after lens insertion, over-refraction was performed using a trial frame to achieve best-distance monocular HCVA with minimal minus over-refraction. If a residual over-refraction was found, CLs were changed to the appropriate power. CL fit was assessed by the unmasked investigator to ensure an acceptable fit before dispensing the CLs for $\sim 1$ week. All dispensed CLs were overlabeled with an "R" or an "L" to mask participants to lens type and differentiate right eye and left eye.

At the end of 1 week of lens wear, participants returned for an assessment visit, during which acuity, binocular vision measurements, and subjective data were collected by masked investigators. A minimum two-night washout period was observed, during which participants wore their habitual corrections before returning for the next fitting visit (Figure 2).

\section{Visual acuity, binocular vision, and stereopsis measurements}

At fitting visits, monocular and binocular HCVA were measured at $6 \mathrm{~m}$ with the Test Chart 2000 Pro, with contrast set to $100 \%$. Near HCVA was measured at $40 \mathrm{~cm}$ with the highcontrast black-on-white ETDRS 2000 Series Logarithmic Visual Acuity Chart. All fitting visit measurements were taken under photopic conditions.

At assessment visits, HCVA and low-contrast visual acuity (LCVA) were measured binocularly at $6 \mathrm{~m}$ with the Test Chart 2000 Pro, with contrast set to $100 \%$ and $10 \%$, respectively. HCVA and LCVA were also measured

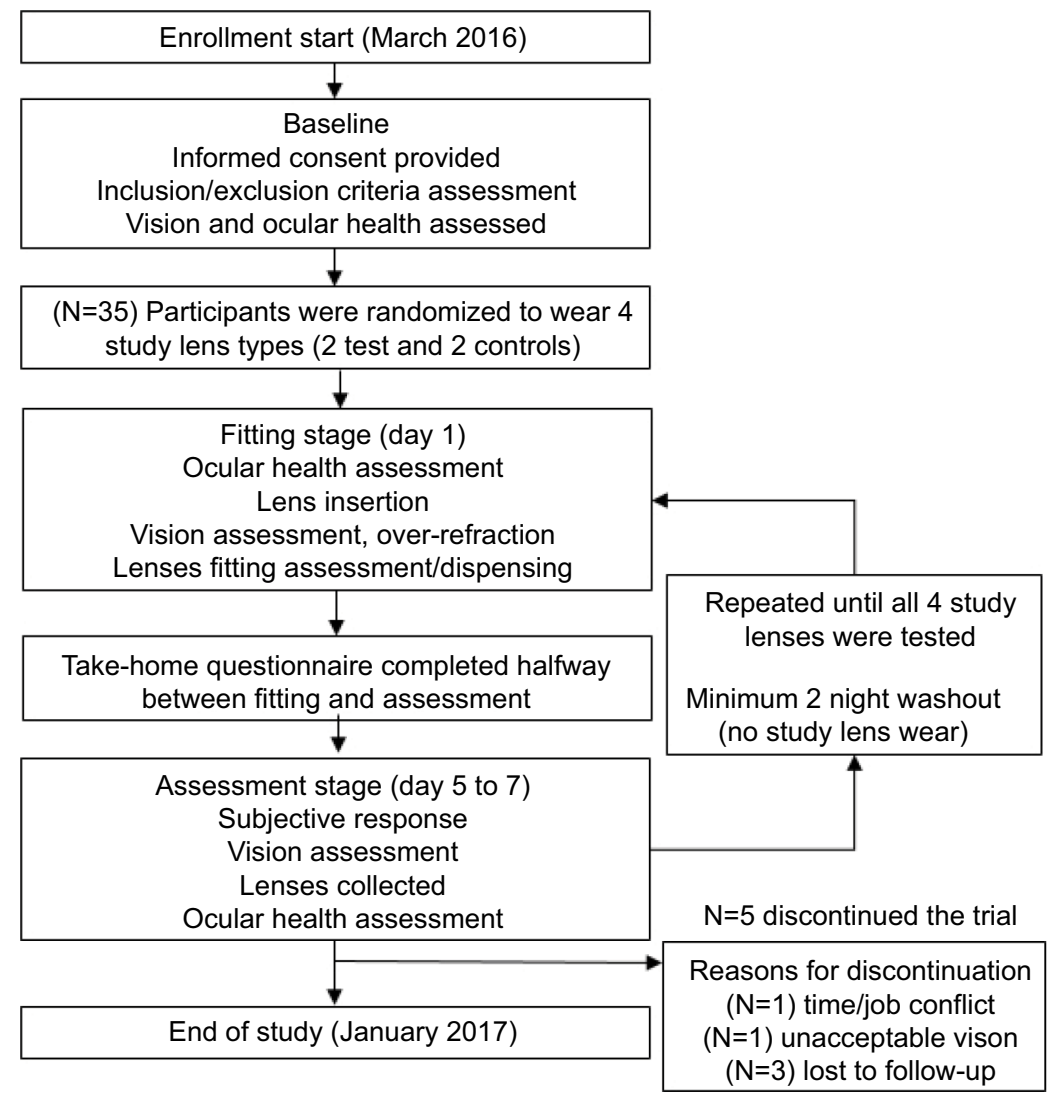

Figure 2 Participant flow chart. 
binocularly at $70 \mathrm{~cm}$, to represent computer distance, and $40 \mathrm{~cm}$ with a high- and low-contrast black-on-white ETDRS 2000 Series Logarithmic Visual Acuity Charts. These charts are designed for $40 \mathrm{~cm}$, so acuity measurements at $70 \mathrm{~cm}$ were converted to equivalent values in $\log$ MAR prior to statistical analysis. Stereopsis, distance and near horizontal phoria, and monocular accommodative facility were measured as per baseline methods. All measurements were taken under photopic conditions. The analysis comprised acuity measurements from assessment visits but not those from fitting visits.

\section{Subjective questionnaire}

The subjective questionnaire encompassed a broad range of visual tasks/demands needed on a daily basis. The questionnaire was administered twice: once as a take-home questionnaire to be filled approximately halfway between the fitting and assessment visit, and once at the assessment visit. The average of responses for the two questionnaires was used for analysis as all responses were well correlated (Pearson's $r=0.763-0.918, p<0.001)$ and a linear mixed model showed that the responses were not significantly different for all variables $(p \geq 0.131)$ except intermediate clarity in the day time $(p=0.027)$. All subjective ratings were based on a numeric rating scale (NRS) scored on a 1-10 interval, in 1-unit steps. Similar questionnaires have been used in our previous assessment of prototype EDOF and commercially available multifocal lenses. ${ }^{22,25-29}$ Questionnaires comprised clarity of vision ( $1=$ blurred, $10=$ clear $)$, and ghosting ( $1=$ none, $10=$ severe) for distance, intermediate, and near distances under day- and night-time conditions. Targeted questions on vision stability under day- and night-time conditions ( 1 = very unstable, $10=$ very stable), driving vision under day- and night-time conditions ( $1=$ blurred, $10=$ clear $)$, night-time haloes $(1=$ not bothersome, $10=$ extremely bothersome $)$, ocular comfort ( $1=$ uncomfortable, $10=$ comfortable $)$, and overall vision satisfaction ( $1=$ not satisfied, $10=$ satisfied $)$ were also included. Frequency of eyestrain symptoms (never/infrequently/sometimes/fairly often/always) was also reported with the following questions: "Do you feel sleepy when reading or doing close work with these lenses?", and "Do you lose concentration when reading or doing close work with these lenses?"

At assessment visits, participants were asked to recall average daily CL wear-time and number of hours per day with acceptable vision. Participants were also asked about their willingness to purchase lenses by answering the following questions with a yes/no response: "Based on vision only, would you buy these lenses?", "If these lenses help slow down the progression of myopia (short-sightedness), would you buy them?"

\section{Data transformation}

To simplify interpretation, the scale for ghosting rating and the scale for night-time haloes have been reversed to be consistent with the other ratings so that a higher rating refers to a better result (i.e., less ghosting and less bothersome haloes). The ghosting variable will, therefore, be referred to as "lack-of-ghosting" and the halo variable will be referred to as "lack-of-haloes". As the stereopsis data were positively skewed, a log transform to the raw data was performed prior to statistical analyses.

\section{Statistical analysis}

A minimum sample of 27 participants was needed to demonstrate a statistically significant paired difference of $1 \pm 1.8$ between lens types for subjective variables with $80 \%$ power and $5 \%$ level of significance. This sample also had $90 \%$ power to detect $0.10 \pm 0.15 \log$ MAR difference in visual acuity between the lens types. All the analyses were performed using SPSS 21 (IBM Corporation, Armonk, NY, USA) and the level of significance was set at 5\%. Post hoc multiple comparisons were adjusted using Bonferroni correction.

Data are summarized as observed means \pm SD for variables measured on an interval scale. All categorical variables are summarized as percentages. For subjective and visual acuity variables, a linear mixed model with subject random intercepts was deployed to test the hypothesis that the prototype lenses performed differently to the controls. The model included lens type as a factor for all variables. For HCVA and LCVA, the model also included test distance as a factor. For all subjective variables, ocular comfort was included as a factor. For clarity of vision and lack of ghosting, test distance and time of day were included as factors. For driving vision and vision stability, time of day was included as a factor.

The interaction of lens type with all other factors was tested. If the interaction of lens type with any other factor was found to be significant, further tests were deployed to determine significance of lens type within sublevels of the interacting factor. In all other instances, the results are generalized for the whole group. This modeling approach was used instead of several paired $t$-tests to avoid inflating the type I error rate. Willingness-to-purchase questions and frequency of eyestrain symptom questions were analyzed using chi-square test. 


\section{Optical performance modeling}

The power profiles of the commercial and the prototype lenses in the distance label powers of $-1.00,-3.00$, and $-6.00 \mathrm{D}$ were measured (NIMO TR1504; Lambda-X) and employed for the theoretical optical performance evaluation in Zemax optical performance evaluation in OpticStudio 17 (Zemax, Kirkland, WA, USA). A schematic model eye was defined $^{30}$ and the vitreous chamber depth was optimized to achieve eyes with refractive errors of $-1.00,-3.00$, and -6.00 D. A CL, specified with the measured power profiles of each lens type, was used to correct the corresponding schematic myopic model eye. Theoretical optical performance was computed at $5 \mathrm{~mm}$ pupil using the ESF and LSF and pupil sampling $1024 \times 1024$. The slope of the ESF and the blur patch size (i.e., background noise) of the LSF were obtained and compared between lenses. A steep slope in the ESF suggests good optical performance, translating to good visual acuity, while an increased blur patch size in the LSF indicates reduced contrast, which may translate to poor subjective visual performance.

\section{Results}

\section{Demographics}

A total of 35 participants commenced the study, and 30 completed the study. Of those who completed the study, $63 \%$ were female, the mean \pm SD age was $24 \pm 5$ years (range 18-33 years), 23\% were Caucasian, $43 \%$ were Asian, and $33 \%$ other ethnicity. Of the five participants who discontinued during the course of the study, three were lost to follow-up, one withdrew because of a time/ job conflict, and one withdrew because of unaccept- able subjective vision with study lenses. Only data from participants who completed all study lenses were included in the analysis. No significant adverse events resulted from lens wear and there were no instances where a lens fit was unacceptable.

\section{Acuities and binocular vision}

HCVA and LCVA results are presented in Figure 3, while binocular vision results are presented in Table 1. The interaction between lens type and test distance was significant for both HCVA and LCVA $(p<0.001)$. MiSight was significantly better than both Prototype 1 and Prototype 2 for binocular HCVA and LCVA at $6 \mathrm{~m}(p \leq 0.006)$. Proclear Multifocal was significantly better than both Prototype 1 and Prototype 2 for binocular LCVA at $6 \mathrm{~m}(p \leq 0.027)$ and better than Prototype 2 for binocular HCVA at $6 \mathrm{~m}(p<$ $0.001)$. Prototype 1 was significantly better than Prototype 2 for binocular LCVA at $6 \mathrm{~m}(p=0.018)$. Lens type was a significant factor for monocular accommodative facility, with participants achieving significantly higher facilities with Prototype 2 and Prototype 1 than with MiSight $(p \leq 0.010)$.

No significant differences between lenses were found for HCVA or LCVA at 70 or $40 \mathrm{~cm}$, stereopsis, or distance or near phoria $(p>0.05)$.

\section{Subjective variables}

Observed ratings for subjective variables that used the 1-10 NRS are shown in Figure 4. Ocular comfort, for which Prototype 1 was rated significantly better than Proclear Multifocal $(p=0.019)$ and both Prototypes 1 and 2 were

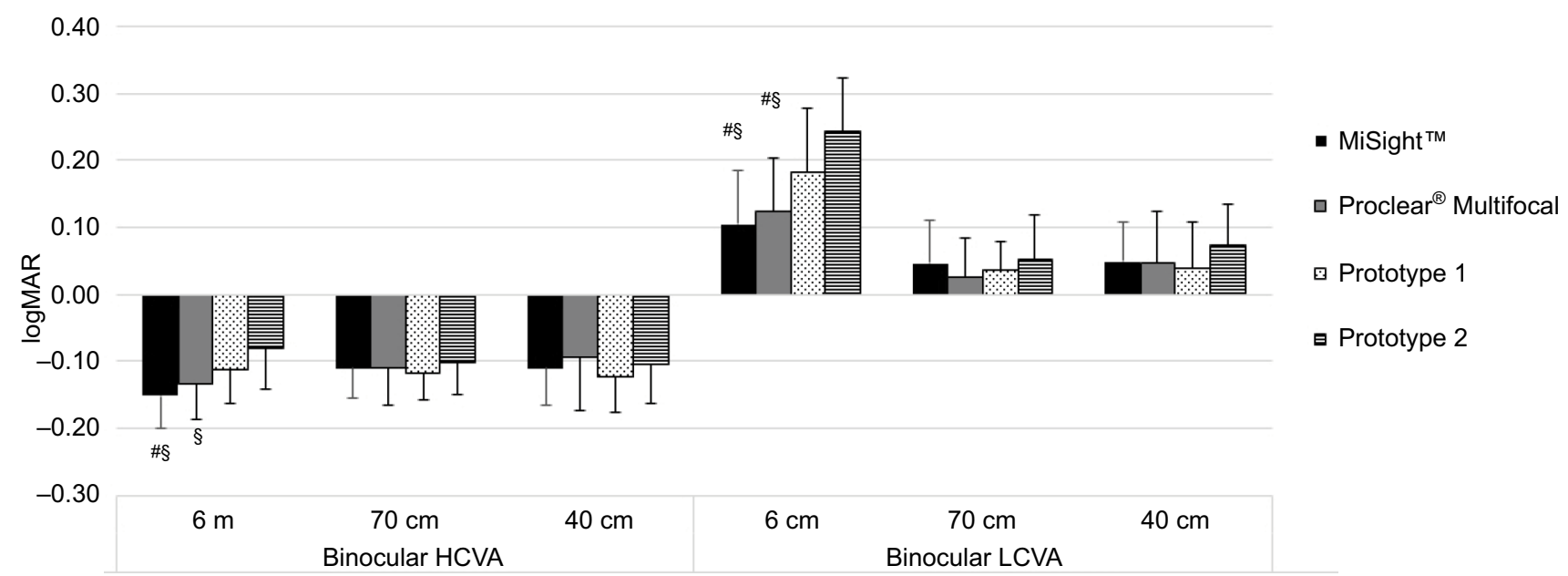

Figure 3 Mean \pm SD Binocular HCVA and LCVA at $6 \mathrm{~m}$, and 70 and $40 \mathrm{~cm}$.

Notes: \#Indicates a significant difference of the MiSight and Proclear control lens to Prototype I ( $p \leq 0.027)$. Indicates a significant difference of the control lens to Prototype $2(p<0.001)$.

Abbreviations: HCVA, high-contrast visual acuity; LCVA, low-contrast visual acuity. 
rated significantly better than MiSight ( $p \leq 0.008$ ), was found to be a significant factor for all subjective NRS variables $(p \leq 0.015)$. Therefore, results for these are also reported as estimated means (mean results calculated assuming average ocular comfort) in Table 2 and in the rest of this section to adjust for the possible effect of comfort.

Lens type was a significant factor for vision clarity, lack of ghosting, overall vision satisfaction, and lack of haloes $(p \leq$ $0.002)$, but not for driving vision or vision stability $(p \geq 0.102)$. Test distance was a significant factor for vision clarity $(p<$ 0.001 ), with higher mean ratings at distance. Time of day was a significant factor for vision clarity, lack of ghosting, driving vision, and vision stability ( $p \leq 0.048$ ), with higher mean ratings in the day time. However, the interactions of test distance and time of day with lens type were not significant $(p>0.05)$.

Table I Mean \pm SD stereopsis, monocular accommodative facility, and distance and near phoria

\begin{tabular}{lllll}
\hline Variable & MiSight $^{\mathrm{TM}}$ & $\begin{array}{l}\text { Proclear }^{\circledR} \\
\text { Multifocal }\end{array}$ & $\begin{array}{l}\text { Prototype } \\
\text { I }\end{array}$ & $\begin{array}{l}\text { Prototype } \\
\mathbf{2}\end{array}$ \\
\hline $\begin{array}{l}\text { Stereopsis } \\
\text { (seconds of arc) }\end{array}$ & $23 \pm \mathrm{II}$ & $24 \pm \mathrm{I} 3$ & $20 \pm 5$ & $22 \pm \mathrm{II}$ \\
$\begin{array}{l}\text { Monocular } \\
\text { accommodative }\end{array}$ & $\mathrm{II} .7 \pm 4.8^{\mathrm{a}, \mathrm{b}}$ & $\mathrm{I} 3.0 \pm 4.8$ & $\mathrm{I} 4.5 \pm 3.9$ & $\mathrm{I} .0 \pm 4.3$ \\
$\begin{array}{l}\text { facility (cycles/ } \\
\text { minute) }\end{array}$ & & & & \\
$\begin{array}{l}\text { Distance phoria } \\
\text { (prism diopters) }\end{array}$ & $-0.6 \pm 2.0$ & $-0.6 \pm 1.0$ & $-0.8 \pm 1.4$ & $-0.9 \pm 1.5$ \\
$\begin{array}{l}\text { Near phoria } \\
\text { (prism diopters) }\end{array}$ & $-2.2 \pm 3.3$ & $-3.2 \pm 4.1$ & $-2.3 \pm 3.3$ & $-2.2 \pm 3.2$ \\
\hline
\end{tabular}

Notes: alndicates a significant difference to Prototype I $(p=0.00 \mathrm{I})$. ' Indicates a significant difference to Prototype $2(p=0.010)$. Negative phoria indicates exophoria.
Prototype 1 was rated significantly higher than both MiSight and Proclear Multifocal for vision clarity, lack of ghosting, and overall vision satisfaction $(p \leq 0.047)$. Prototype 1 was also rated significantly higher than MiSight for lack of haloes $(p<0.001)$, and higher than Prototype 2 for overall vision clarity $(p=0.021)$.

Participants rated Prototype 2 significantly higher than both MiSight and Proclear Multifocal for lack of ghosting $(p<0.001)$. Prototype 2 was also rated significantly higher than MiSight for lack of haloes $(p=0.003)$.

Proclear Multifocal was rated significantly higher than MiSight for lack of ghosting $(p<0.001)$. MiSight was not rated significantly higher than any other lens for any subjective variable.

Frequency of eyestrain symptoms is shown in Figure 5. There were no differences in frequency of eyestrain symptoms between the test and control lenses ( $p \geq 0.441)$.

Participants wore Prototypes 1 and 2 for significantly longer each day on average than MiSight ( $p \leq 0.001)$, and also found their vision acceptable for significantly longer each day when wearing Prototypes 1 and 2 compared to MiSight $(p \leq 0.001)$. These data are shown in Table 3.

There was a significant difference between lens types for willingness to purchase ( $p=0.012$ ) with Prototype 1 having the highest willingness-to-purchase rate (Figure 6). A higher proportion of participants would purchase all lens types if myopia progression was slowed but there was no significant difference between lens types in this scenario $(p=0.248)$.

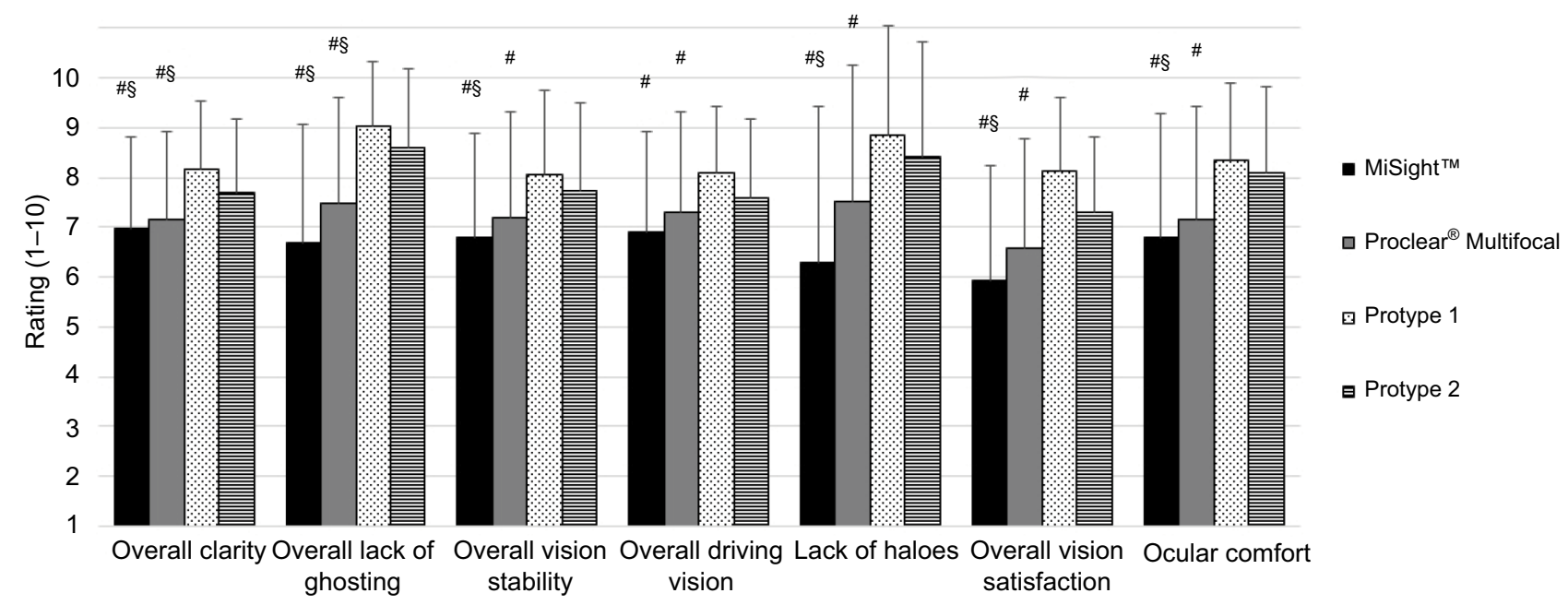

Figure 4 Observed mean \pm SD ratings for subjective variables.

Notes: A higher rating indicates a better outcome. \#Indicates a significant difference of the control lens to Prototype I ( $p \leq 0.039)$. ${ }^{\S}$ Indicates a significant difference of the control lens to Prototype $2(p \leq 0.008)$. 


\section{Optical performance modeling}

There were distinctive differences between the ESFs and LSFs of the commercial lenses and the prototype lenses (Figure 7).

While the MiSight and the Proclear Multifocal Distance lenses showed a steep slope in the ESF that occurred within the central 10 and $20 \mu \mathrm{m}$ of the retina, respectively, the prototype lenses showed a more gradual slope within the central $40 \mu \mathrm{m}$ of the retina.

Comparison of the LSFs showed that the blur patch sizes of the MiSight and the Proclear Multifocal Distance lenses were about $160 \mu \mathrm{m}$ across the central retina, but the blur patch sizes of the prototype lenses were about $90 \mu \mathrm{m}$ across the central retina.

The ESFs and LSFs remained constant with changes in refractive error.

Table 2 Estimated mean ratings $(95 \% \mathrm{Cl})$ for subjective variables after adjusting for ocular comfort ratings

\begin{tabular}{lllll}
\hline Variable & MiSight $^{\mathrm{TM}}$ & $\begin{array}{l}\text { Proclear }^{\circledR} \\
\text { Multifocal }^{\text {Prototype I }}\end{array}$ & $\begin{array}{l}\text { Prototype } \\
\mathbf{2}\end{array}$ \\
\hline Overall clarity & 7.3 & 7.3 & 7.9 & 7.5 \\
& $(6.9-7.6)^{\mathrm{a}}$ & $(7.0-7.7)^{\mathrm{a}}$ & $(7.5-8.2)$ & $(7.2-7.9)$ \\
Overall lack of & 6.9 & 7.6 & 8.9 & 8.5 \\
ghosting & $(6.5-7.3)^{\mathrm{a}, \mathrm{b}}$ & $(7.2-8.0)^{\mathrm{a}, \mathrm{b}}$ & $(8.4-9.3)$ & $(8.1-8.9)$ \\
Overall vision & 7.3 & 7.5 & 7.6 & 7.5 \\
stability & $(6.8-7.7)$ & $(7.0-7.9)$ & $(7.2-8.0)$ & $(7.0-7.9)$ \\
Overall driving & 7.1 & 7.5 & 7.8 & 7.5 \\
vision & $(6.5-7.6)$ & $(7.0-8.1)$ & $(7.2-8.3)$ & $(6.9-8.0)$ \\
Lack of haloes & 6.5 & 7.7 & 8.6 & 8.3 \\
& $(5.6-7.5)^{\mathrm{a}, \mathrm{b}}$ & $(6.7-8.6)$ & $(7.7-9.6)$ & $(7.4-9.2)$ \\
Overall vision & 6.4 & 6.8 & 7.7 & 7.0 \\
satisfaction & $(5.9-6.9)^{\mathrm{a}}$ & $(6.4-7.3)^{\mathrm{a}}$ & $(7.2-8.2)$ & $(6.5-7.5)$ \\
\hline
\end{tabular}

Notes: Indicates a significant difference of the control lens to Prototype I $(p \leq 0.047)$. bIndicates a significant difference of the control lens to Prototype $2(p \leq 0.003)$.

\section{Discussion}

With myopia an increasing worldwide problem, multifocal soft CLs have emerged as one of the strategies to reduce myopia progression in children. Past research on myopia control using multifocal soft CLs generally reports a $25 \%-50 \%$ reduction in myopia progression compared to single vision. ${ }^{13,14,16,17}$ However, the visual performance of myopic control soft CLs also tends to be reduced, ${ }^{22,23}$ which may lead to decreased compliance with lens wear and reduction in the efficacy of myopia control. ${ }^{16,21}$ Hence, a multifocal soft CL that is effective in myopia control while also maintaining good visual performance is desirable.

In the present study, we compared the wearability of four different CL designs that have shown efficacy in myopia control. MiSight and center-distance Proclear Multifocal are commercially available CLs with reported myopia control efficacy of $36 \%-59 \%$ over 10 months to 3 years for MiSight ${ }^{17,18}$ and $51 \%$ over 2 years for Proclear Multifocal. ${ }^{13}$ The other two test lenses are prototype designs. The efficacy of the test lenses

Table 3 Mean \pm SD average daily wear time and average daily acceptable vision time

\begin{tabular}{lllll}
\hline Variable & MiSight $^{\mathrm{TM}}$ & $\begin{array}{l}\text { Proclear }^{\circledR} \\
\text { Multifocal }\end{array}$ & $\begin{array}{l}\text { Prototype } \\
\text { I Prototype }\end{array}$ & $\mathbf{2}$ \\
\hline $\begin{array}{l}\text { Average daily wear } \\
\text { time (hours) }\end{array}$ & $7.6 \pm 3.4^{\mathrm{a}, \mathrm{b}}$ & $8.5 \pm 3.7$ & $9.3 \pm 2.8$ & $9.5 \pm 2.9$ \\
$\begin{array}{l}\text { Average daily } \\
\text { acceptable vision } \\
\text { time (hours) }\end{array}$ & $5.5 \pm 3.6^{\mathrm{a}, \mathrm{b}}$ & $6.9 \pm 3.9$ & $7.9 \pm 2.7$ & $7.8 \pm 3.0$ \\
\hline
\end{tabular}

Notes: andicates a significant difference of the control lens to Prototype I ( $p \leq 0.00 \mathrm{I})$. Indicates a significant difference of the control lens to Prototype $2(p \leq 0.00 \mathrm{I})$.

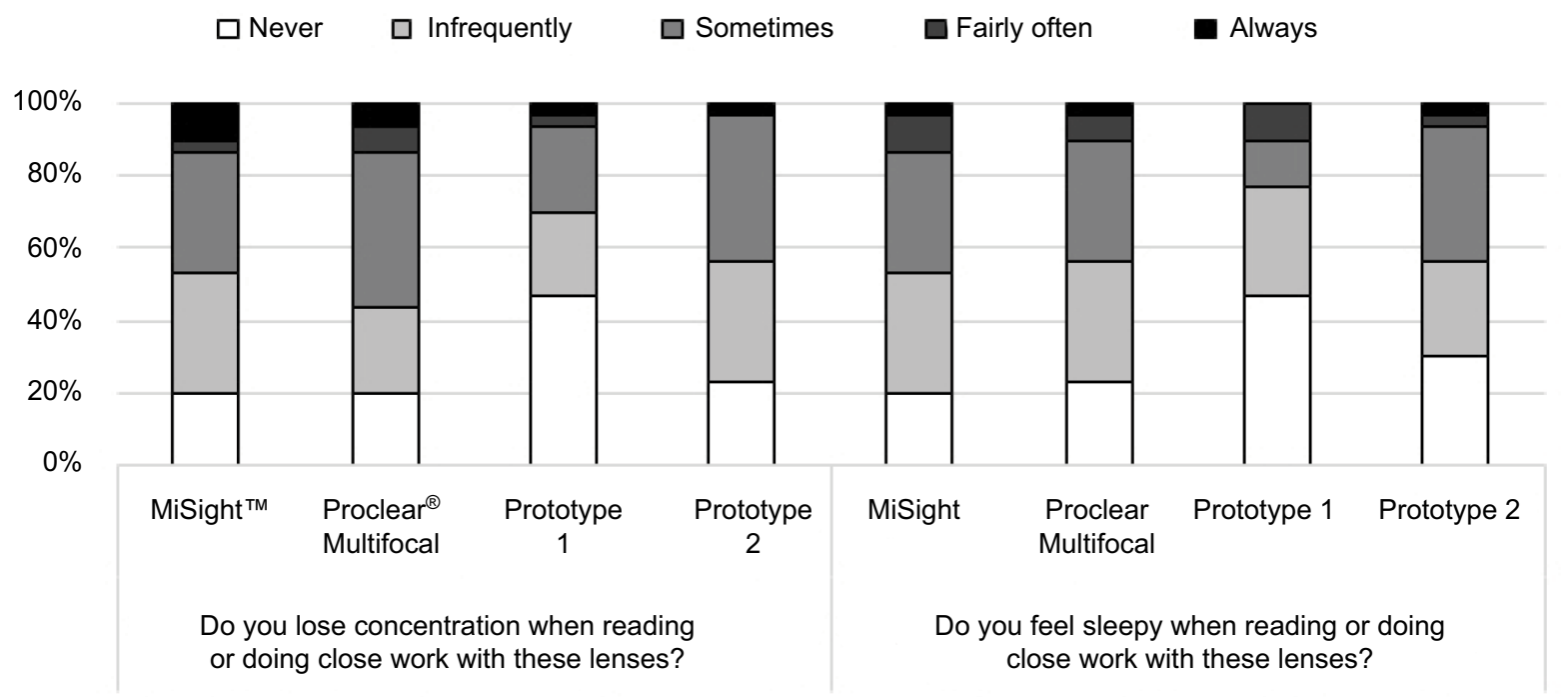

Figure 5 Frequency of eyestrain symptoms.

Note: No significant differences were found between lens types $(p \geq 0.44 \mathrm{I})$. 
100\% Would buy $\square$ Would buy if lens slows myopia progression

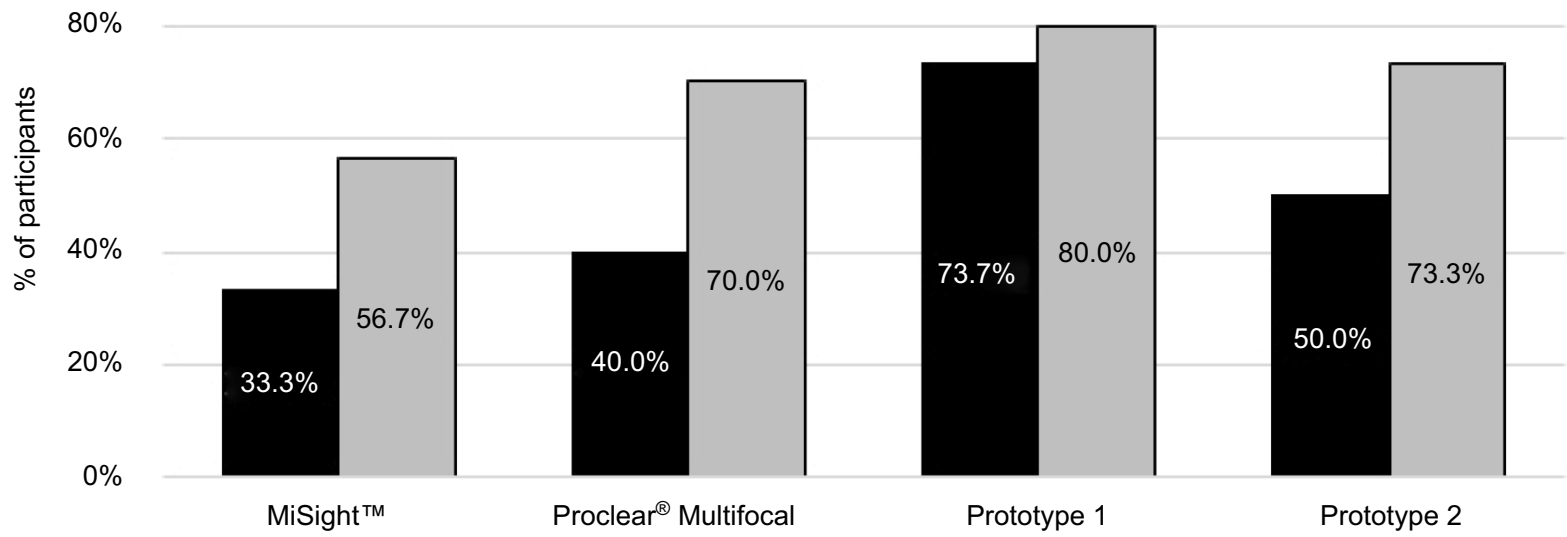

Figure 6 Willingness to purchase lenses.

Notes: There was a significant difference between lens types for willingness to purchase $(p=0.012)$ but not for willingness to purchase if lens slows myopia progression ( $p=0.248$ ).

(Prototypes 1 and 2) is currently being trialed in China, and the 12-month results from this trial have been recently reported: Prototypes 1 and 2 showed adjusted mean myopia control of $27 \%$ and $33 \%$, respectively, compared to the control. ${ }^{21}$ The differences in efficacy between MiSight, Proclear Multifocal, Prototype 1, and Prototype 2 could be due to a number of factors such as differences in lens design, participant age group, study design, baseline refraction, parental myopia, ethnicity, and/or peripheral refraction profiles. However, to explore these variables is clearly beyond the scope of the current study.

In terms of visual acuity, the control lenses MiSight and center-distance Proclear Multifocal provided participants in our study with excellent HCVA and LCVA at all distances, even exceeding the visual acuities reported by Anstice and Phillips, Walline et al, and Fedtke et al. ${ }^{13,17,22}$ However, this was not reflected in participants' subjective responses, where they preferred the prototypes, particularly Prototype 1, in the majority of subjective variables tested. This suggests that participants found their quality of vision with MiSight and Proclear Multifocal compromised by factors that do not necessarily affect their ability to resolve letters, such as ghosting and/or haloes. Indeed, the subjective variables that showed the greatest differences between the commercial and prototype lenses were those related to ghosting and haloes, problems that arise from simultaneous imaging CLs because of the production of multiple defocused images on the retina. ${ }^{22,31}$

These results support past work indicating that subjective assessments are more sensitive than objective assessments in determining success and patient satisfaction with multifocal CLs. ${ }^{25,32}$ The discrepancy between objective and subjective results in our study is also in line with the studies by
Kollbaum et al and Fedtke et al, which found that participants attained good HCVA with MiSight and Proclear Multifocal, but they rated subjective visual performance significantly lower than with habitual or single-vision correction. ${ }^{22,23}$

To further understand the differences observed in previous studies and the current study, optical performance, as described by the ESF and the LSF, was computed and compared between the commercial and the prototype lenses. The steeper slope of the ESF and the greater blur patch size of the LSF with the commercial lenses when compared to the prototype lenses are in line with the visual performance results of this study, that is, the better visual acuity, but lower subjective visual performance results with the commercial lenses. This agreement between objective and subjective performance measures also suggests that the ESF and LSFs may be valuable features when optimizing visual performance of CLs.

Our monocular accommodative facility results showed that MiSight caused the lowest facility, while Proclear Multifocal and Prototypes 1 and 2 were no different to each other. Ozkan et al found that monocular accommodative facilities with four multifocal CLs, including Proclear Multifocal Distance, were lower than that of a single-vision $\mathrm{CL}$ in a group of 18-25-year-old myopic CL wearers. ${ }^{33}$ We can deduce that Proclear Multifocal and Prototypes 1 and 2 cause some disruption to accommodative facility when compared to the single-vision CL, but MiSight causes the most disruption. Despite this, Anstice and Phillips reported that MiSight was able to stimulate accommodation for near vision because of its large central zone. ${ }^{17}$ However, these authors tested lag of accommodation with MiSight in one eye, while the other eye wore a single-vision distance lens. It is possible that wearing 

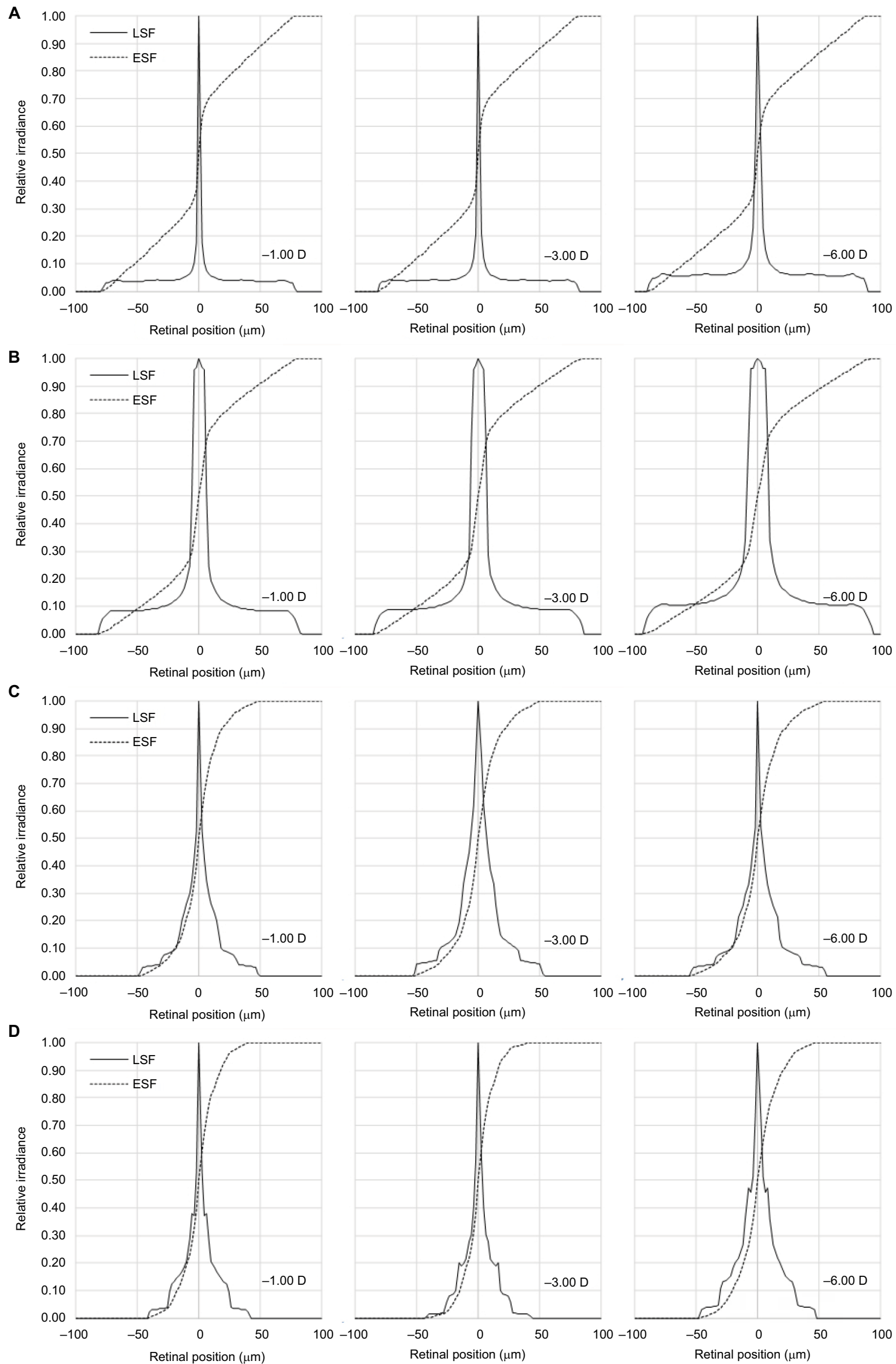

Figure 7 Geometric LSF and ESF for all study lenses in powers $-1.00,-3.00$, and $-6.00 \mathrm{D}$.

Note: (A) MiSight ${ }^{\mathrm{TM}}$. (B) Proclear $^{\circledR}$ Multifocal. (C) Prototype I. (D) Prototype 2.

Abbreviations: ESF, edge spread function; LSF, line spread function. 
MiSight binocularly would have caused more disruption to the accommodative system. Ozkan et al also reported an average monocular accommodative facility with center-distance Proclear Multifocal of $16.1 \pm 8.5$ cycles/minute..$^{33}$ The lower mean monocular accommodative facility in our study may be due to a more difficult target, as they used a 6/12 Snellen "E" letter, while we used a $0.2 \log$ MAR (6/9) paragraph of text. Our distance and near phoria measurements for Proclear Multifocal lenses are in agreement with previous observations. ${ }^{33}$

In terms of wearing time, Prototypes 1 and 2 had the longest average daily wearing time of the study lenses, with the average wearing time being $>9$ hours. MiSight had the shortest average daily wearing time of 7.6 hours. Anstice and Phillips reported in their study an average daily wearing time with MiSight lenses of 11.9 hours/day at 2 weeks, which increased to 13.15 hours/ day at 20 weeks. ${ }^{17}$ However, an 8 hour per day minimum wear time was imposed in their study, compared to a 6 hour per day minimum in the current study. Furthermore, participants in the study of Anstice and Phillips wore a single-vision distance lens in one eye, potentially improving visual performance. We acknowledge that our current study was limited in its duration, and patient adaptation may result in increased wearing time of MiSight lenses compared to our study; however, we would also expect increases in wearing time with other lenses.

We chose to dispense center-distance Proclear Multifocal as a daily disposable, despite normally being a monthly daily-wear lens. This was to avoid potential comfort issues with reused lenses and lens care solution, to improve convenience for participants, and to maintain robustness of the masking and randomization scheme. With respect to ocular comfort, it was interesting to note that the observed means for ocular comfort ratings mirrored the ratings of most of the other variables. When the means were adjusted for comfort, the effect of lens type became nonsignificant for vision stability and driving vision, but remained significant for vision clarity, ghosting, haloes, and overall vision satisfaction. From this, it appears as though there was at least some subconscious influence of comfort on vision or vice versa. This phenomenon has been investigated and reported previously ${ }^{34,35}$ Regardless, even after adjusting for comfort, participants in this study rated the prototypes, particularly Prototype 1, higher than MiSight and Proclear Multifocal in a number of variables.

In terms of comparing the visual performance of MiSight against center-distance Proclear Multifocal, the results from the current study showed no differences between the two for all variables except Proclear Multifocal, which was rated as having less overall ghosting compared to MiSight. Though they did not directly compare the two, Fedtke et al also found higher mean ratings for their subjective variables with Proclear Multifocal than with MiSight. ${ }^{22}$ Overall, this suggests that center-distance Proclear Multifocal, a lens primarily used for correcting presbyopia, is at least slightly better tolerated by non-presbyopic myopes than MiSight. ${ }^{13,17}$

Our results showed that willingness-to-purchase values for all the study lenses increased if they were to slow myopia progression, suggesting that myopes are willing to tolerate some level of visual compromise in order to reduce their risk of myopia progression, at least in the short term. It is unknown whether they would have the same tolerances over a longer period of time, such as months to years, but it is probable that lenses with the least visual compromise would have the best chance of being worn successfully in the long term. Further study is required to show how well these lenses are tolerated over a longer period.

A limitation of the current study was that the questionnaire was not validated. However, similar questions were asked in our previous trials..$^{22,25-29}$ Furthermore, as this was a randomized, crossover study, any bias would remain constant throughout all administrations of the questionnaire.

Myopia progression is of greatest concern in children; therefore, results obtained from the 18-35 age group used in the current study may not be entirely representative of children. As the current study focused on the wearability, particularly the subjective performance of the test lenses, young adults were chosen as the tasks may have been difficult for a young child to comprehend and respond accurately. Performing the same study in myopic children would be useful to confirm the results obtained from young adults.

\section{Conclusions}

Prototypes 1 and 2 had better subjective visual performance in numerous variables and were better tolerated by myopes compared to the commercial soft CLs currently suggested for slowing myopia progression. In summary, these new CL designs have both the potential for myopia control while still maintaining good visual acuity and favorable subjective acceptance.

\section{Acknowledgments}

The authors would like to acknowledge the clinical team (Ms K. Wagenfeuhr, Ms E. Robertson, and Ms B. Ludlow) and the database management team (Dr T. Naduvilath and Ms $\mathrm{K}$. Laarakkers) for their invaluable support to run this trial at the CRTC, Brien Holden Vision Institute, Sydney, NSW, Australia. The authors would also like to acknowledge Dr J. Flanagan for reviewing the manuscript. This project is entirely funded by the Brien Holden Vision Institute. The Brien Holden Vision Institute has proprietary interests in the 
intellectual property governing extended-depth-of-focus contact lenses (Patent application number WO2014059465 A1).

\section{Disclosure}

All authors are employed by the Brien Holden Vision Institute. The authors report no other conflicts of interest in this work.

\section{References}

1. Holden BA, Fricke TR, Wilson DA, et al. Global prevalence of myopia and high myopia and temporal trends from 2000 through 2050. Ophthalmology. 2016;123:1036-1042.

2. Grossniklaus HE, Green WR. Pathologic findings in pathologic myopia. Retina. 1992;12:127-133.

3. Saw SM, Gazzard G, Shih-Yen EC, Chua WH. Myopia and associated pathological complications. Ophthal Physiol Opt. 2005;25:381-391.

4. Holden BA, Sankaridurg P, Smith E, Aller T, Jong M, He M. Myopia, an underrated global challenge to vision: where the current data takes us on myopia control. Eye. 2014;28:142-146.

5. Jonas JB, Xu L. Histological changes of high axial myopia. Eye. 2014:28:113-117.

6. Kitaguchi Y, Bessho K, Yamaguchi T, Nakazawa N, Mihashi T, Fujikado T. In vivo measurements of cone photoreceptor spacing in myopic eyes from images obtained by an adaptive optics fundus camera. Jpn J Ophthalmol. 2007;51:456-461.

7. Chui TY, Song H, Burns SA. Individual variations in human cone photoreceptor packing density: variations with refractive error. Invest Ophthalmol Vis Sci. 2008;49:4579-4587.

8. Coletta NJ, Watson T. Effect of myopia on visual acuity measured with laser interference fringes. Vision Res. 2006;46:636-651.

9. Flores-Moreno I, Ruiz-Medrano J, Duker JS, Ruiz-Moreno JM. The relationship between retinal and choroidal thickness and visual acuity in highly myopic eyes. Br J Ophthalmol. 2013;97:1010-1013.

10. Nishida Y, Fujiwara T, Imamura Y, Lima L, Kurosaka D, Spaide RF. Choroidal thickness and visual acuity in highly myopic eyes. Retina. 2012;32(7):1229-1236.

11. Walline JJ, Gaume A, Jones LA, et al. Benefits of contact lens wear for children and teens. Eye Contact Lens. 2007;33:317-321.

12. Walline JJ, Long S, Zadnik K. Daily disposable contact lens wear in myopic children. Optom Vis Sci. 2004;81:254-258.

13. Walline JJ, Greiner KL, McVey ME, Jones-Jordan LA. Multifocal contact lens myopia control. Optom Vis Sci. 2013;90(11):1207-1214.

14. Sankaridurg P, Holden BA, Smith E, et al. Decrease in rate of myopia progression with a contact lens designed to reduce relative peripheral hyperopia: one-year results. Invest Ophthalmol Vis Sci. 2011;52:9362-9367.

15. Ruiz-Alcocer J. Analysis of the power profile of a new soft contact lens for myopia progression. J Optom. 2017;10(4):266-268.

16. Lam CSY, Tang WC, Tse DY, Tang YY, To CH. Defocus incorporated soft contact (DISC) lens slows myopia progression in Hong Kong Chinese school-children: a 2-year randomised clinical trial. Br J Ophthalmol. 2014;98:40-45.

17. Anstice NS, Phillips JR. Effect of dual-focus soft contact lens wear on axial myopia progression in children. Ophthalmology. 2011;118: $1152-1161$
18. Chamberlain P. 3-year effectiveness of a dual-focus 1 day soft contact lens for myopia control. Paper presented at: British Contact Lens Association (BCLA) Clinical Conference \& Exhibition; June 10, 2017; Liverpool, UK.

19. Aller TA, Liu M, Wildsoer CF. Myopia control with bifocal contact lenses: a randomized clinical trial. Optom Vis Sci. 2016;93: 344-352.

20. Bakaraju RC, Xu P, Chen X, et al. Extended depth of focus (EDOF) contact lenses can slow the rate of myopia progression. Invest Ophthalmol Vis Sci. 2015;56(7):Abstract 1728.

21. Sankaridurg P, Bakaraju RC, Morgan J, et al. Novel contact lenses designed to slow progress of myopia: 12 month results. Poster presented at: Association for Research in Vision and Ophthalmology (ARVO); May 7-11, 2017; Baltimore, USA. Abstract 2391.

22. Fedtke C, Bakaraju RC, Ehrmann K, Chung J, Thomas V, Holden BA. Visual performance of single vision and multifocal contact lenses in non-presbyopic myopic eyes. Cont Lens Anterior Eye. 2016;39(1):38-46.

23. Kollbaum PS, Jansen ME, Tan J, Meyer DM, Rickert ME. Vision performance with a contact lens designed to slow myopia progression. Optom Vis Sci. 2013;90(3):205-214.

24. Kang P, McAlinden C, Wildsoet CF. Effects of multifocal soft contact lenses used to slow myopia progression on quality of vision in young adults. Acta Ophthalmol. 2017;95(1):e43-e53.

25. Diec J, Tilia D, Naduvilath T, Bakaraju RC. Predicting short-term performance of multifocal contact lenses. Eye Contact Lens. 2016;43(6):340-345.

26. Sha J, Bakaraju RC, Tilia D, et al. Short-term visual performance of soft multifocal contact lenses for presbyopia. Arq Bras Oftalmol. 2016;79: 73-77.

27. Tilia D, Bakaraju RC, Chung J, et al. Short-term visual performance of novel extended depth-of-focus contact lenses. Optom Vis Sci. 2016;93(4): 435-444.

28. Tilia D, Munro A, Chung J, et al. Short-term comparison between extended depth-of-focus prototype contact lenses and a commerciallyavailable center-near multifocal. J Optom. 2017;10:14-25.

29. Bakaraju RC, Tilia D, Sha J, et al. Extended depth of focus contact lenses vs. two commercial multifocals: part 2. Visual performance after 1 week of lens wear. J Optom. 2018;11(1):21-32.

30. Bakaraju RC, Ehrmann K, Ho A, Papas E. Inherent ocular spherical aberration and multifocal contact lens optical performance. Optom Vis Sci. 2010;87(12):1009-1022.

31. Kollbaum PS, Dietmeier BM, Jensen ME, Rickert ME. Quantification of ghosting produced with presbyopic contact lens correction. Eye Contact Lens. 2012;38(4):252-259.

32. Papas EB, Decenzo-Verbeten T, Fonn D, et al. Utility of short-term evaluation of presbyopic contact lens performance. Eye Contact Lens. 2009;35(3):144-148.

33. Ozkan J, Fedtke C, Chung J, Thomas V, Bakaraju RC. Short-term adaptation of accommodative responses in myopes fitted with multifocal contact lenses. Eye Contact Lens. 2016;0:1-8.

34. Papas EB, Chan E, Sarian L, Tan J. Does the quality of vision affect the perception of ocular discomfort? Invest Ophthalmol Vis Sci. 2003;44:3694.

35. Rao SB, Simpson TL. Influence of vision on ocular comfort ratings. Optom Vis Sci. 2016;93(8):793-800.
Clinical Optometry

\section{Publish your work in this journal}

Clinical Optometry is an international, peer-reviewed, open access journal publishing original research, basic science, clinical and epidemiological studies, reviews and evaluations on clinical optometry. All aspects of patient care are addressed within the journal as well as the practice of optometry including economic and business analyses. Basic and clinical Submit your manuscript here: https://www.dovepress.com/clinical-optometry-journal

\section{Dovepress}

research papers are published that cover all aspects of optics, refraction and its application to the theory and practice of optometry. The manuscript management system is completely online and includes a very quick and fair peer-review system, which is all easy to use. Visit http://www.dovepress. com/testimonials.php to read real quotes from published authors. 